\title{
Criptococosis cerebral en paciente inmunocompetente: reporte de caso y revisión de la literatura
}

\author{
Cerebral cryptococosis in an immunocompetent patient: case report and literature review \\ Hollman Armando Ávila-Coy, ${ }^{*}$ María José López-Mora, ${ }^{\ddagger}$ Óscar Bernal-Pacheco§ \\ * Médico residente Neurología, Universidad Militar Nueva Granada. Bogotá, Colombia. https://orcid.org/0000-0002-6036-9905. \\ ‡ Médica Infectóloga, Especialista Infectología. Hospital Militar Central. Bogotá, Colombia. https://orcid.org/0000-0001-9865-9097. \\ $\S$ Médico Neurólogo, Subespecialista en Movimientos Anormales. Hospital Militar Central. Bogotá, Colombia. https://orcid.org/0000-0001-6435-3137.
}

Citar como: Ávila-Coy HA, López-Mora MJ, Bernal-Pacheco O. Criptococosis cerebral en paciente inmunocompetente: reporte de caso y revisión de la literatura. Neurol Neurocir Psiquiatr. 2021; 49 (2): 69-72. https://dx.doi.org/10.35366/103354

\section{RESUMEN}

La criptococosis en sistema nervioso central es una infección infrecuente en pacientes inmunocompetentes, el complejo Cryptococcus, del cual forman parte Cryptococcus neoformans y Cryptococcus gattii, causa infección de inicio en pulmón y posteriormente infección en sistema nervioso central; es más frecuente en pacientes inmunocompetentes Cryptococcus gattii y en pacientes inmunocomprometidos Cryptococcus neoformans, teniendo como presentación clínica relevante cefalea por aumento de presión intracraneal; el presente artículo reporta un caso clínico de un hombre de 46 años, inmunocompetente, que asiste por cefalea con signos de alarma que son: primer episodio de cefalea de características de aumento de presión intracraneal que no cede con analgesia, realizando estudios complementarios, siendo estos neuroimágenes y punción lumbar con identificación con reacción en cadena de la polimerasa en líquido cefalorraquídeo, en los cuales se identifica infección en sistema nervioso central por Cryptococcus gattii y durante estancia hospitalaria se identifica nódulo pulmonar con criptococoma, por lo cual se realiza lobectomía, y se ordena tratamiento con anfotericina B y fluconazol.

Palabras clave: Criptococosis cerebral, neuroinfección, inmunocompetente, sistema nervioso central, Cryptococcus gattii.
ABSTRACT

Cryptococcosis in the central nervous system is an infrequent infection in immunocompetent patients. The Cryptococcus complex of which Cryptococcus neoformans and Cryptococcus gattii is a part, cause infection that starts in the lung and later causes infection in the central nervous system; being more frequent in immunocompetent patients Cryptococcus gattii and in immunocompromised patients Cryptococcus neoformans; having as a relevant clinical presentation headache due to increased intracranial pressure; this article reports a clinical case of a 46-year-old male, immunocompetent, who attends due to headache with warning signs, the warning signs being: first episode of headache with characteristics of increased intracranial pressure, which does not subside with analgesia, performing complementary studies, these being neuroimaging and lumbar puncture with identification with polymerase chain reaction in cerebrospinal fluid, in which infection in the central nervous system by Cryptococcus gattii is identified and during hospital stay a pulmonary nodule with cryptococoma is identified, for which lobectomy was performed, and treatment with amphotericin B and fluconazole was ordered.

Keywords: Cerebral cryptococcosis, neuroinfection, immunocompetent, central nervous system, Cryptococcus gattii.

\section{INTRODUCCIÓN}

Cryptococcus neoformans y Cryptococcus gattii son una levadura que contiene una cápsula de polisacáridos, que metaboliza la urea y las catecolaminas, y por lo general se transmite por la inhalación de excremento de aves, principalmente palomas; la criptococosis es una enfermedad fúngica que tiene como vía de contagio la inhalación y posterior infección pulmonar, puede diseminarse al sistema nervioso central (SNC) y causar meningitis o meningoencefalitis. ${ }^{1-3}$

Por lo general, la infección inicial es de localización pulmonar y a partir de ésta se disemina a otros órganos con especial preferencia por el sistema nervioso central, donde

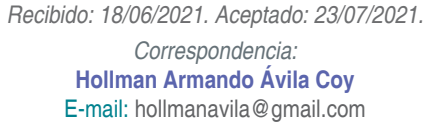


causa meningoencefalitis y muy rara vez lesiones focalizadas granulomatosas conocidas como criptococomas. ${ }^{4}$ Esta infección afecta principalmente dos sitios: el pulmón y el SNC, aunque puede afectar otros órganos, como riñón, próstata y huesos. De los sitios blanco, afecta mayormente al SNC, el cual representa el sitio donde se genera en 50\% de los casos debido al tropismo del hongo. ${ }^{5}$ La mayoría de los casos ocurren en huéspedes inmunodeprimidos, incluidos pacientes con $\mathrm{VIH} / \mathrm{SIDA}$, pacientes que reciben fármacos inmunosupresores y receptores de trasplantes de órganos sólidos. Sin embargo, la criptococosis también ocurre en individuos inmunocompetentes. ${ }^{2,6}$ La criptococosis en sistema nervioso central es una infección infrecuente en pacientes inmunocompetentes; la presentación de este tipo de criptococosis puede ser de dos formas:

1. Quistes en corteza cerebral con reacción meníngea granulomatosa.

2. Granulomas en sustancia blanca llamados criptococomas. ${ }^{1,6,7}$

Se ha reportado que en pacientes inmunocompetentes existe una relación hombre:mujer de 2:1, mientras que en pacientes inmunocomprometidos hay una relación de hasta $11: 1.8,9$

En la mayoría de los casos documentados, el tratamiento de la infección por $\mathrm{C}$. gattii del SNC requiere un manejo agresivo de la presión intracraneal elevada junto con la terapia antimicótica estándar. ${ }^{10-12}$

El tratamiento se divide en tres fases: fase de inducción anfotericina B deoxicolato $0.7 \mathrm{mg} / \mathrm{kg} /$ día intravenosa (IV), o anfotericina B liposomal 3-4 mg/ $/ \mathrm{kg} /$ día +5 fluocitosina $100 \mathrm{mg} / \mathrm{kg} /$ día vía oral (VO) dividida en cuatro dosis durante dos semanas (hasta seis semanas en caso de presencia de criptococomas); seguido de una fase de consolidación para la cual se debe tener cultivo en líquido cefalorraquídeo negativo fluconazol 400-800 mg cada día por vía oral por ocho semanas; y una última fase de mantenimiento fluconazol 200-400 mg/día vía oral por mínimo 12 meses. ${ }^{13,14}$

\section{MÉTODOS}

Se describe un caso clínico confirmado mediante la realización de cultivo en líquido cefalorraquídeo de criptococosis en sistema nervioso central por Cryptococcus gattii en un paciente inmunocompetente, en el Servicio de Neurología del Hospital Militar Central Bogotá (Colombia); asimismo, se realiza una revisión de la literatura actualizada disponible acerca de criptococosis en sistema nervioso central en paciente inmunocompetente; la información fue tomada de la Historia Clínica del Hospital Militar Central, previa aprobación mediante consentimiento informado por parte del paciente.

\section{CASO CLÍNICO}

Hombre de 46 años de edad, diestro, que tiene como profesión técnico en estructuras de aeronaves, que ingresa por cefalea de cinco días, holocraneana, de inicio progresivo, tipo punzada, alcanza máxima intensidad 8/10, sin foto ni fonofobia, sin nauseas ni emesis, que aumenta con cambios de posición y maniobras de Valsalva, que cede temporalmente con acetaminofén y diclofenaco, permite conciliar sueño, en algunas ocasiones la cefalea despierta, sin antecedentes de importancia para la enfermedad actual; al examen físico y neurológico dentro de límites normales, se toman estudios de neuroimagen como parte de la atención integral, los resultados de la tomografía craneal se muestran en la Figura 1.

Se complementa estudio con resonancia magnética nuclear con contraste, la cual se muestra en la Figura 2. Y dados los hallazgos en probable relación con aumento de presión intracraneal, se realiza punción lumbar encontrando los siguientes resultados:

- PA: $40 \mathrm{cmH}_{2} \mathrm{O}$ PC: $12 \mathrm{cmH}_{2} \mathrm{O}$.

- Aspecto turbio.

- Gram negativo para microorganismos.

Figura 1:

Múltiples lesiones focales hipodensas localizadas en las regiones nucleobasales, probablemente de etiología infecciosa-inflamatoria.
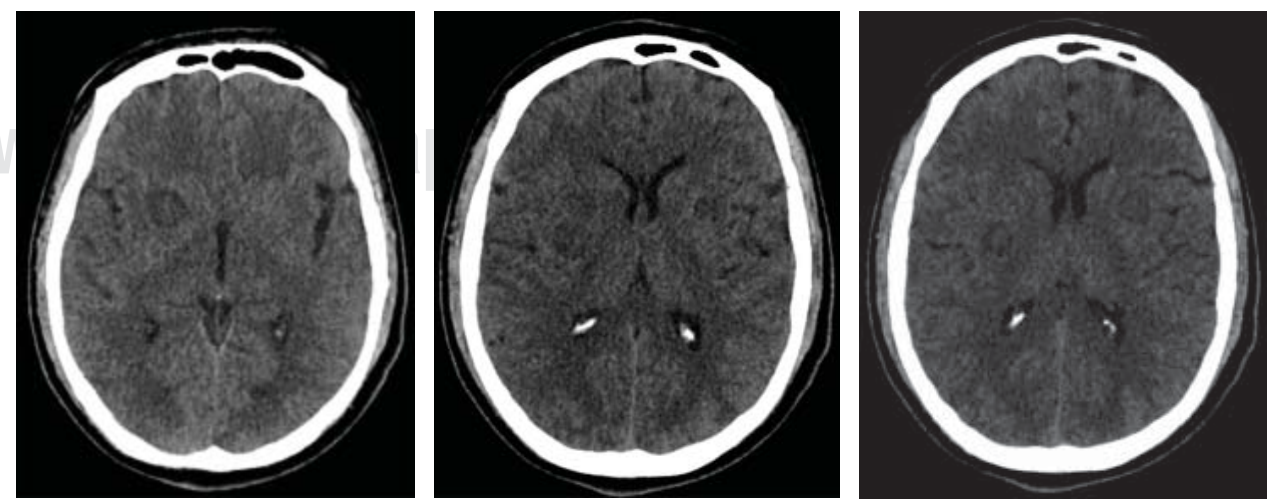

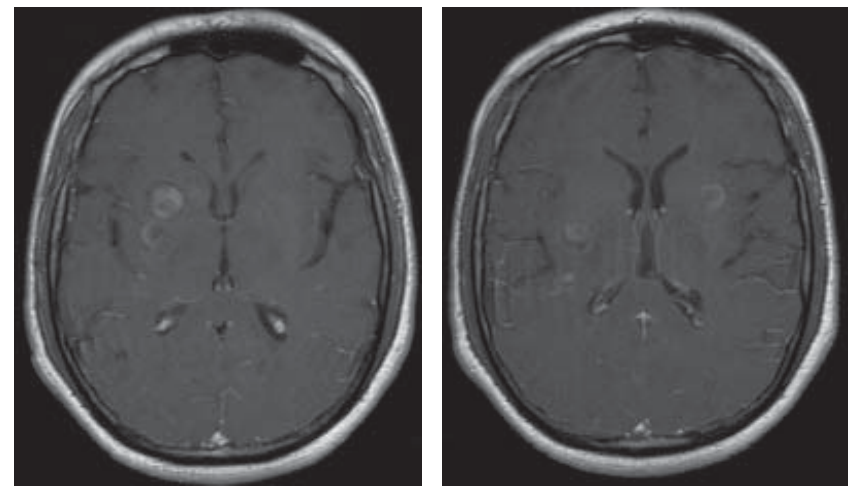

Figura 2: Lesiones focales redondeadas, bien definidas, nucleobasales bilaterales de baja intensidad de señal en secuencias T1, alta señal en secuencias sensibles al líquido, algunas presentan ligero edema perilesional y realce central grueso irregular tras la administración de contraste endovenoso asociado a realce leptomeníngeo.

- Proteínas totales 117.4.

- Glucosa LCR 4.17 Suero 93 IG 0.04.

- Leucocitos 150, 27 segmentados 20 linfocitos, monocitos 3.

- Hematíes 48 crenados 10.

- VDRL No reactivo.

- Film array positivo Cryptococcus neoformans/gattii.

- Gene Xpert Negativo TBC.

- Tinta china positiva para levaduras encapsuladas.

Aislando en cultivo de líquido cefalorraquídeo: Cryptococcus gattii, sensible a anfotericina y fluconazol (Tabla 1).

Dado su cuadro clínico y aislamiento microbiológico, se decide iniciar manejo con anfotericina B liposomal y fluconazol, en fase de inducción durante seis semanas de tratamiento (contexto criptococomas); durante hospitalización se realizan estudios de inmunidad: VIH (negativo) en tres oportunidades, y estudios de inmunidad primaria dentro de límites normales.

Con cultivos de control en líquido cefalorraquídeo negativos, y con adecuada evolución clínica por ausencia de cefalea, por lo que se da egreso hospitalario en fase de consolidación con fluconazol 400 mg c/día.

Sin embargo, paciente reconsulta al mes del egreso por presentar nuevamente cefalea de las mismas características, para lo cual se toman nuevas neuroimágenes (Figura 3).

Punción lumbar:

- Presión de apertura: $32 \mathrm{~cm}$ de $\mathrm{H}_{2} \mathrm{O}$.

- Presión de cierre: $18 \mathrm{~cm}$ de $\mathrm{H}_{2} \mathrm{O}$.

- Gram negativo.

- Tinta china negativo.

- Índice de glucosa 0.48 .
- VDRL: no reactivo.

- Proteínas: 79.

- Leucocitos: 2 (linfocitos).

- FilmArray negativo.

Se decide hospitalizar para estudios de extensión, como una tomografía de tórax de alta resolución, evidenciándose nódulo pulmonar localizado en el segmento superior del lóbulo inferior izquierdo con diámetros de $29 \times 17 \mathrm{~mm}$, para lo cual se realiza biopsia que muestra criptococoma en lóbulo inferior izquierdo y se realiza lobectomía inferior izquierda con los siguientes hallazgos de estudios de patología: parénquima pulmonar con extensa inflamación crónica granulomatosa con presencia de microorganismos de tipo Cryptococcus gattii, siendo este el foco de infección primario; por lo anterior, en junta multidisciplinaria se decide reiniciar terapia de inducción con anfotericina B y flucitosina por seis semanas, y nuevamente cultivo de líquido cefalorraquídeo al finalizar dicha fase, el cual resulta negativo, para lo cual se da continuidad a tratamiento con fluconazol en fase de consolidación y mantenimiento, con mejoría clínica y paraclínica del paciente con seguimiento por la consulta externa, con buen curso de evolución.

\section{REVISIÓN DE LA LITERATURA Y DISCUSIÓN}

La infección por Cryptococcus en sistema nervioso central en pacientes inmunocompetentes es poco frecuente, Cryptococcus gattii tiene mayor incidencia en este tipo de pacientes, causando mayor morbilidad por presentación de criptococomas y mayor tiempo de tratamiento; el diagnós-

Tabla 1: Resumen de resultados

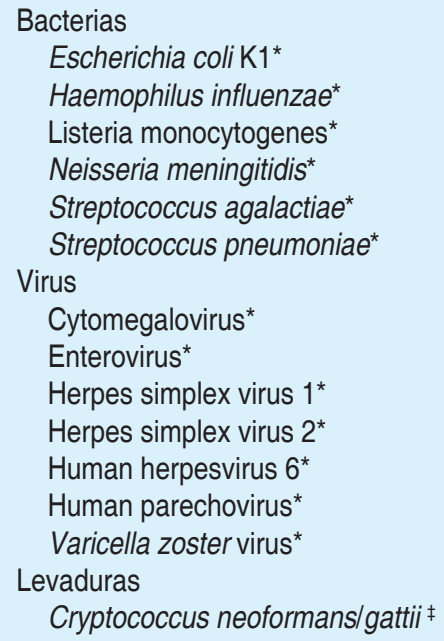

* No detectado, ${ }^{\ddagger}$ Detectado. 

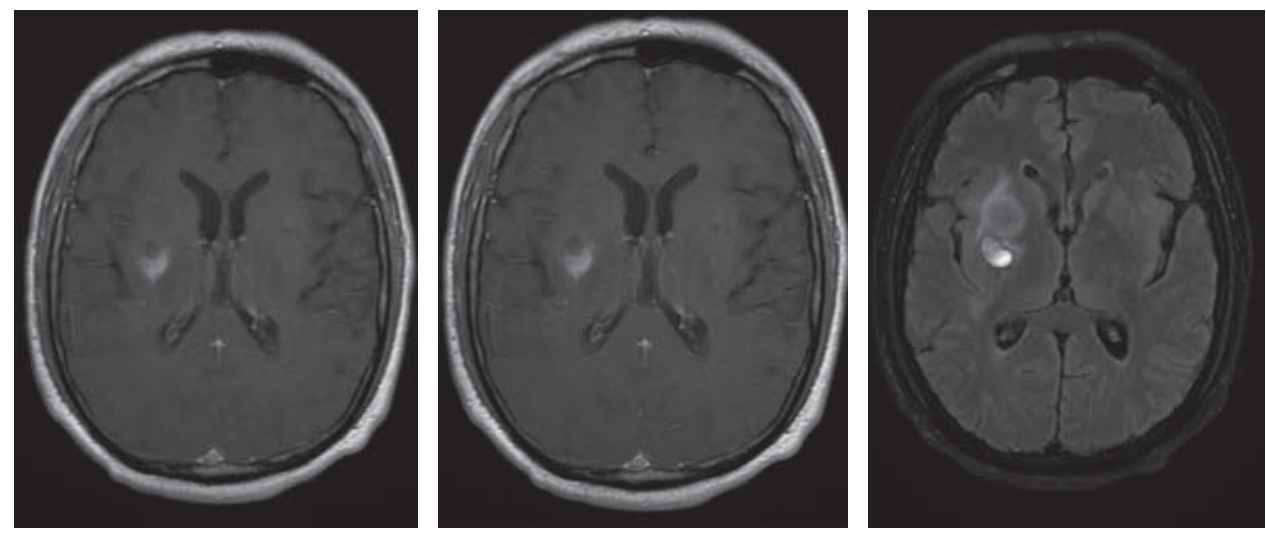

Figura 3:

Imagen de resonancia magnética: mejoría imagenológica significativa compatible con respuesta a tratamientos establecidos, persistiendo de menor tamaño con reducción de la reacción de edema vasogénico.

tico clínico es difícil de establecer por el inicio subagudo de los síntomas y la manifestación poco específica en pacientes inmunocompetentes, la mayoría de los casos cursa como una meningitis subaguda o crónica caracterizada por cefalea, náuseas, vómitos, fiebre, alteración de la conciencia, signos de hipertensión intracraneana y signos meníngeos y en casos más complejos se presentan criptococomas, el diagnóstico es por medio de una punción lumbar y la tinta china la herramienta que dará el diagnóstico definitivo; se encontrará levadura en aproximadamente $75 \%$ de los pacientes con $\mathrm{VIH}$ y $50 \%$ de los inmunocompetentes, y el cultivo nos permite conocer el tipo de Cryptococcus causante de la infección. En el caso de nuestro paciente, se encontró como foco primario de la infección un criptococoma en lóbulo inferior de pulmón izquierdo, y especial interés en la presencia de criptococomas en parénquima cerebral en paciente inmunocompetente, lo cual conlleva a un mayor tiempo de tratamiento. ${ }^{7,13,15}$

Conflicto de intereses: Los autores declaran no tener conflicto de intereses para el presente escrito.

Financiamiento: Para la realización del presente artículo no se requirió apoyo económico, al ser una descripción de caso clínico.

\section{REFERENCIAS}

1. Franco-Paredes C, Womack T, Bohlmeyer T, Sellers B, Hays A, Patel $\mathrm{K}$ et al. Management of Cryptococcus gattii meningoencephalitis. Lancet Infect Dis. 2015; 15 (3): 348-355. doi: 10.1016/S14733099(14)70945-4.

2. Galnares-Olalde JA, Loza-Jalil S. Cryptococcal meningitis in an immunocompetent patient: case report and literature review. Revista Médica del Hospital General de México. 2014; 77 (3): 137-141.

3. Tay Z, Lara R, Velasco C, Gutiérrez A. Parasitología médica. 6a ed., Méndez Editores, 2002.

4. Rana AQ, Saeed U, Rana AN. Role of environmental factors in Cryptococcal meningitis in immunocompetent individuals. Acta Neurol Belg. 2014; 114: 75-76.

5. Maziarz EK, Perfect JR. Cryptococcosis. Infect Dis Clin North Am. 2016; 30 (1): 179-206.

6. Chen SCA, Meyer W, Sorrell TC. Cryptococcus gattii infections. Clin Microbiol Rev. 2014; 27(4): 980-1024.

7. Pérez-Veitia JF. Cryptococcal meningoencephalitis: a case report. Rev Mex Neuroci. 2017; 18 (4): 80-85.

8. Day JN, Chau TTH, Wolbers M, Mai PP, Dung NT, Mai NH et al. Combination antifungal therapy for Cryptococcal meningitis. N Engl J Med. 2013; 368: 1291-130222.

9. Jarrín-Maisincho K, Cordovez-Navas M, Vera-Pérez E. Criptococomas cerebrales en un paciente inmunocompetente: reporte de un caso. MedPre. 2012; 2 (1): 23-33.

10. Martin-Blondel G, Ysebaert L. Disseminated Cryptococcosis. N Engl J Med. 2014; 370: 1741

11. Barquero AM. Meningitis por Cryptococcus neoformans. Revista Medica de Costa Rica y Centroamérica LXXIII. 2016; 619; 205-208.

12. Cortés J, Kral A, Wilson G. Cryptococcosis in Hospital Carlos Van Buren, Valparaiso: a clinical serie. Rev Chilena Infectol. 2018; 35 (4): 420-423.

13. Trejo-Espino AW, Ramírez-Izcoa A, Alvarado-Rivera S, Carlos Godoy-Mejía, Renato Valenzuela-Castillo Cryptococcus neoformans meningoencephalitis in a malnourished teenager. Acta Med Peru. 2016; 33 (3): 232-235

14. Messina FA, Maiolo E. Therapeutic alternatives meningeal cryptococcosis. Buenos Aires. 2015; 23 (88): 25-32.

15. Gomez-Pelaez GW. Risk factors of cryptococcosis in patients with HIV in the hospital of infectology "Dr. José Daniel Rodríguez Maridueña" in the period 2015-2016. Dom Cien. ISSN: 2477-8818. 2019; 5 (1): 736-750. 